\title{
Diagnostic Study of Lithium-Rich Cathode Materials at Primary and Sub-Primary Particle Level by Using Chemical-Sensitive STEM Tomography, Aberration- Corrected Imaging and EELS
}

\author{
Ruoqian Lin'1, Xiao-Qing Yang ${ }^{2}$ and Huolin L. Xin ${ }^{2}$ \\ 1. Chemistry Division, Brookhaven National Laboratory \\ 2. Department of Physics and Astronomy, University of California Irvine
}

With the increasing demand of electric vehicles, great effort has been invested into the development of novel high energy density electrode materials for lithium-ion batteries. Lithium-rich layered transition metal oxides have been regarded as promising candidates and attracted great attention in the past decade. With extra lithium stored in the material matrix, much higher specific capacity can be provided than conventional layered cathode materials such as $\mathrm{LiCoO}_{2}$. In the process of lithium extraction, not only cation redox, but also anion redox is activated and contribute to the super high capacity. Unfortunately, the low columbic efficiency of first charge/discharge cycle and voltage fading of lithium-rich cathode materials, which are highly correlated with irreversible oxygen redox activity and chemical/structural degradation, still prohibit their practical application in electric vehicles at current stage. $[1,2]$

By utilizing newly developed chemical-sensitive scanning transmission electron microscopic (STEM) tomography and aberration-corrected imaging technique, the results of the investigation of structural changes of $\mathrm{Li}_{1.2} \mathrm{Ni}_{0.15} \mathrm{Mn}_{0.1} \mathrm{Co}_{0.55} \mathrm{O}_{2}$ (LR-NMC) [1] and $\mathrm{Li}_{2} \mathrm{Ru}_{0.5} \mathrm{Mn}_{0.5} \mathrm{O}_{3}$ (LRMO) [2] will be reported. The results regarding the correlation between the chemical component and structural/chemical evolution irreversibility of lithium-rich cathode materials will also be discussed. STEM tomography is used to three-dimensionally resolve the internal pore structure and its development with sub-nanometer resolution. For example, as shown in Figure 1, after extensive electrochemical cycling, it is found that isolated nano pores are formed in LR-NMC materials, while LRMO material is degraded to sponge-like nanoporous structure with countless sub-nanometer pores. Our results show that the structural degradation in lithium-rich cathode materials is correlated with chemical segregation of the electrode materials at two drastically different length scale: single-digit nanometer and sub-micron length scales. Severe surface structure degradation and transition metal segregation are observed in LRMO materials after extensive charge/discharge cycling. Our results provide a direct evidence the surface passivation plays an important role to determine the degradation mechanism and degradation pathway in lithiumrich cathode materials.

The results of using newly developed chemical-sensitive scanning transmission electron microscopic (STEM) tomography, aberration-corrected imaging, and STEM-EELS to study structural changes of electrode materials for Li-ion battery will be reported (Fig. 2). Some initial results regarding the structural irreversibility and chemical segregation of layer-layer compounds relating to their composition will be discussed as an example. Our results show that upon charge/discharge cycles, it is possible to use STEM tomography to three-dimensionally resolve the internal pore structure and its development with sub-nanometer resolution. This structural degradation is correlated with chemical segregation of the electrode materials at two drastically different length scale - single-digit nanometer and sub-micron length scales. Using our experimental results as a direct input for our high-throughput ab initio calculations, we are able to show that the solubility of transition metals in a specific solid phase can be used as an important parameter in predicting the structural reversibility of transition metal 
cathode materials [3].

References:

[1] E Hu et al., Nature Energy 3 (2018), p. 680.

[2] R Lin, et al., Nature Communications, in press.

[3] The authors acknowledge support from the Assistant Secretary for Energy Efficiency and Renewable Energy, Vehicle Technology Office of the U.S. DOE through the Advanced Battery Materials Research (BMR) Program, including Battery500 Consortium under Contract DE-SC0012704, the Center for Functional Nanomaterials of Brookhaven National Laboratory was supported by the US Department of Energy, Office of Science, Office of Basic Energy Sciences, under contract no. DE-SC0012704, and the U.S. Department of Energy's Office of Energy Efficiency and Renewable Energy (EERE) under the Award Number: DE-EE0008444.

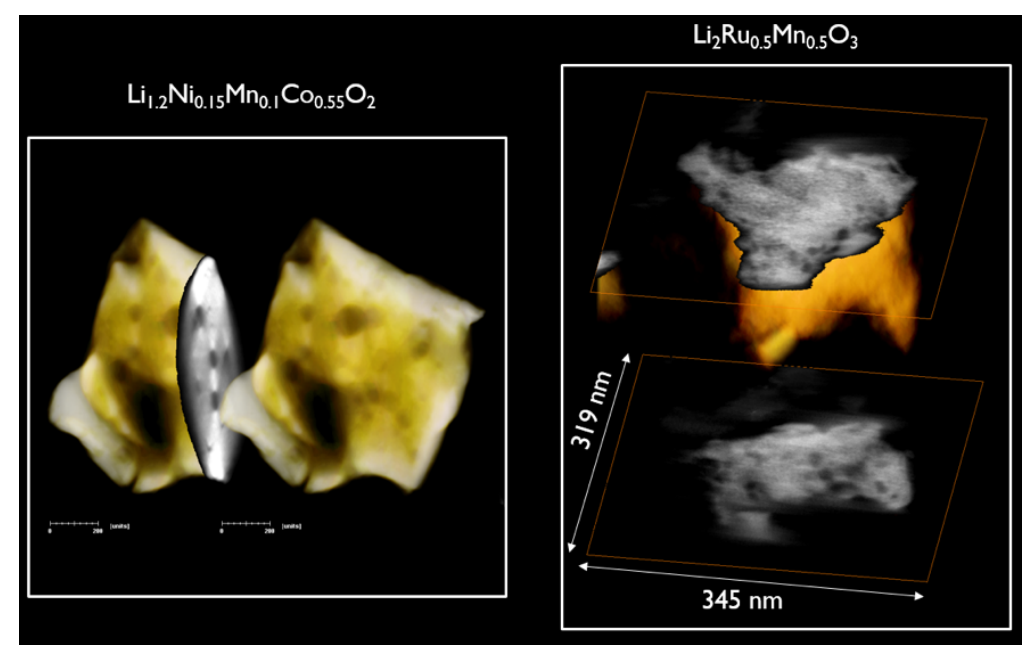

Figure 1. Three-dimensional tomography of $\mathrm{Li}_{1.2} \mathrm{Ni}_{0.15} \mathrm{Mn}_{0.1} \mathrm{Co}_{0.55} \mathrm{O}_{2}(\mathrm{LR}-\mathrm{NMC})$ and $\mathrm{Li}_{2} \mathrm{Ru}_{0.5} \mathrm{Mn}_{0.5} \mathrm{O}_{3}$ (LRMO) material after electrochemical cycling. Left: isolated nano pores are formed in $\mathrm{Li}_{1.2} \mathrm{Ni}_{0.15} \mathrm{Mn}_{0.1} \mathrm{Co}_{0.55} \mathrm{O}_{2}$. Right: sponge-like nanoporous structure is formed in $\mathrm{Li}_{2} \mathrm{Ru}_{0.5} \mathrm{Mn}_{0.5} \mathrm{O}_{3}$.

a

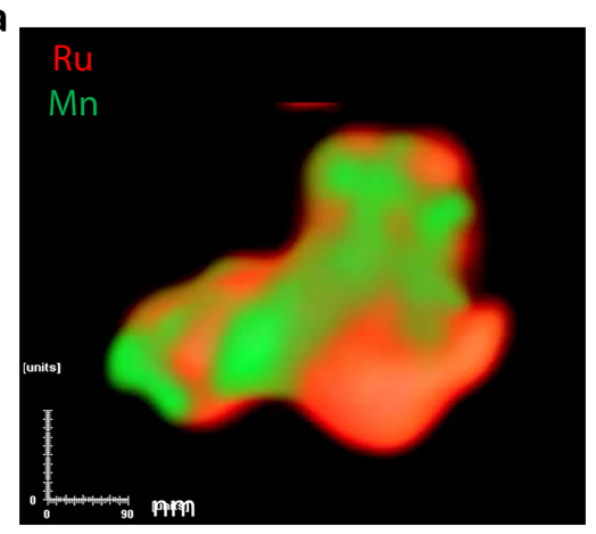

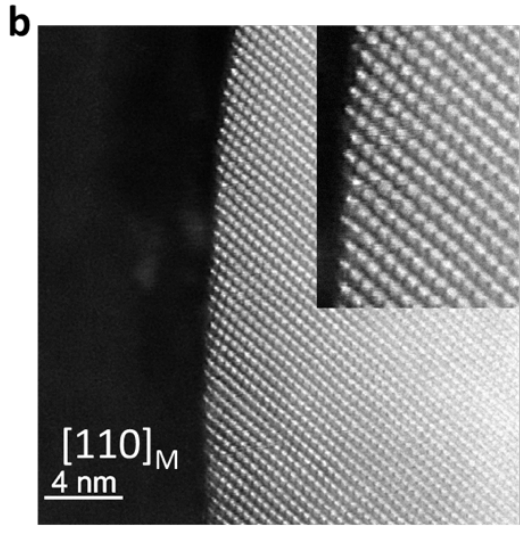

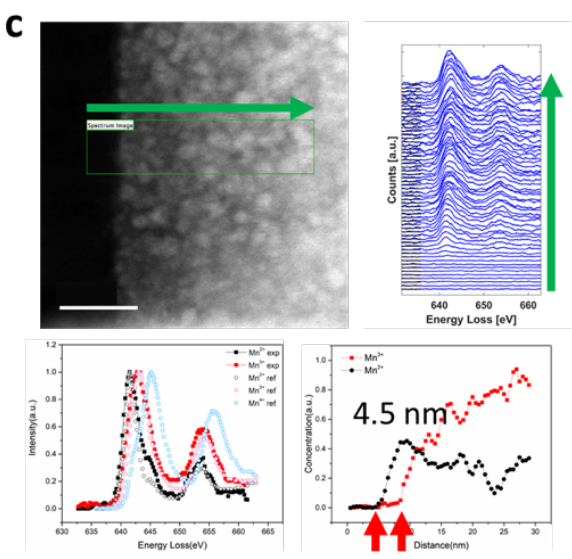

Figure 2. (a) Chemically sensitive tomographic imaging of $\mathrm{Li}_{2} \mathrm{Ru}_{0.5} \mathrm{Mn}_{0.5} \mathrm{O}_{3}$ (LRMO) after extended cycling showing the chemical segregation at the primary particle level. (b) Aberration-corrected ADFSTEM imaging of the pristine LRMO. (c) STEM-EELS analysis of the surface rock-salt reconstruction on cycled LRMO. 